\title{
Steering Timing Prediction in a Driving Simulator Task*
}

\author{
Lucian Gheorghe, Ricardo Chavarriaga, and José del R. Millán IEEE
}

\begin{abstract}
In this paper we present the preliminary results of a pioneering attempt to predict the timing of steering actions in a driving task from non-invasive EEG measurements. The experiment took place with the subjects driving a car at a constant speed on a simulated highway in a driving simulator. The EEG activity was analyzed during periods of straight driving and during lane change actions. Classifiers were built on the signals recorded over the motor areas for straight and pre-steering periods. The onset of the steering actions was detected on average $811 \mathrm{~ms}$ before the action with a $74.6 \%$ true positive rate.
\end{abstract}

\section{INTRODUCTION}

Brain-computer interfaces (BCI) provide means of interaction by decoding brain signals correlated with specific tasks or cognitive states [1]. We are trying to develop a BCI specially tailored for facilitating the interactions between drivers and intelligent cars. The cognitive state of the driver or the intentions for future actions could be used to create a more intuitive vehicle interface, leading to less stress and an improved driving experience. Arousal level prediction based on electroencephalogram (EEG) and electrooculogram (EOG) has been vastly investigated [2], [3], as well as attention level detection, both in real and simulated driving environments [4]. At the same time the automobile industry has made big steps towards the implementation of smart cars that could interpret the environment, provide feedback to the driver and, if needed, control the vehicle [5]. As cars become more intelligent the interaction with the user may increase (to provide more feedback, or suggest potential maneuvers). Efforts have been made to evaluate precisely the drivers' workload in order to facilitate information delivery from the vehicle [6]. Our philosophy is to decode the drivers' brain activity related to driving, and to use these results in order to align the actions of the autonomous vehicle with the driver's intention. For example, being able to accurately predict future actions of the driver would enable the vehicle to adjust, if needed, its dynamics or position in order to facilitate those actions. In such a scenario the vehicle could provide support without explicit feedback or interference, thus greatly improving the feeling of easiness and comfort in control that the driver has. Several preliminary studies aimed at detecting

*This study was supported by Nissan Motor Co. Ltd., and carried out under the "Research on Brain Machine Interface for Drivers" project.

L. Gheorghe, R. Chavarriaga, and J. d. R. Millán are with the Defitech Foundation Chair in Non-Invasive Brain-Machine Interface, Center for Neuroprosthetics, École Polytechnique Fédérale de Lausanne (EPFL), Switzerland.

L. Gheorghe is also from Mobility Services Laboratory, Nissan Research Center, Nissan Motor Co., LTD., Atsugi, Japan (e-mail: lucian@mail.nissan.co.jp). movement intention showed encouraging results [7]. On the other hand, few attempts have been made to predict drivers' motion while driving. Haufe et al. build a system to predict the timing of braking when the car in front slows down [8]. In their research, the response generated by the visual cue of the brake lamps in front and also the motor cortex activity were considered simultaneously. One of the first reports of neural correlates of movement was made by Kornhuber and Deecke back in 1965 [9] showing a slow cortical potential (SCP) appearing $1.5 \mathrm{~s}$ before movement. Libet et al. made a deep analysis of these potentials proving the presence of preparatory brain activity beginning $1 \mathrm{~s}$ before the onset of movement [10][11]. While most of the previous studies have been performed with simplified protocols, this study aims at detecting steering action movements from non-invasive EEG measurements in a natural driving task performed in a realistic driving simulator. Since SCPs are difficult to be recorded even in controlled environments this study is taking quite a challenge in performing the analysis on more natural environment recordings.

\section{METHODS}

\section{A. Experimental Protocol}

A simple but realistic driving simulator was used for this experiment. The layout was close to a small size vehicle using a real automobile electrical seat in order to accommodate the subject in a comfortable driving position. To reduce possible electrical artifacts, electrical force feedback steering systems have been avoided. Instead a passive system based on springs and fluid damping was used to generate a realistic steering feeling.

The driving scene simulates a two lane highway in Switzerland. Six subjects with normal or corrected to normal vision were instructed to drive at constant speed (approx. 120 $\mathrm{km} / \mathrm{h}$ ) and, at certain parts of the course, to do a lane change to the left followed by one to the right. The timing of the lane changes was self paced. No other vehicles were present in the scenario.

Steering and pedal positions, vehicle dynamics and the position of the vehicle in space were recorded at a sampling rate of $256 \mathrm{~Hz}$. Another computer recorded 64 EEG channels placed according to the 10/20 extended standard at $2048 \mathrm{~Hz}$ and down sampled at $256 \mathrm{~Hz}$. The two recordings were synchronized by a hardware trigger. The recording was split in 5 sessions of 12 min recorded the same day. At the subject's request shorter or larger breaks were taken between the sessions with the goal of keeping low fatigue and high concentration levels throughout all the sessions. In order to adapt to the driving simulator visual field and to the controls, a 
30 min trial driving session was performed before the experiment by all subjects. At that time the driving simulator sickness tendencies were also evaluated with all the subjects and no subject showed sickness symptoms.

A

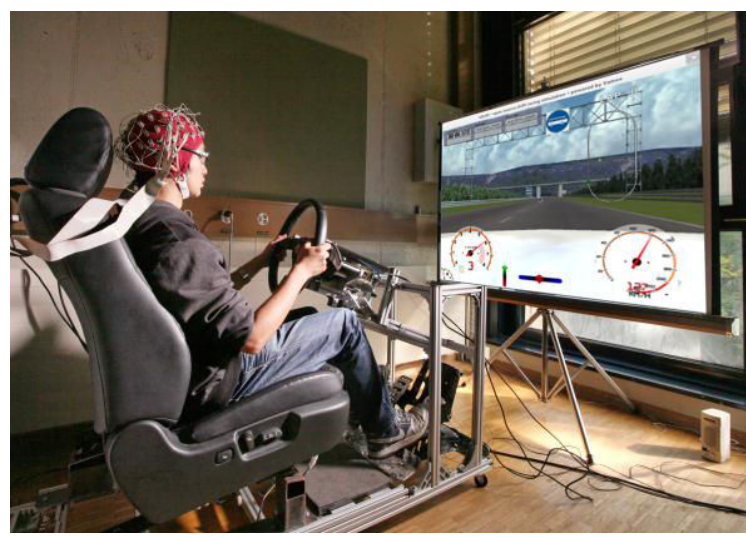

B

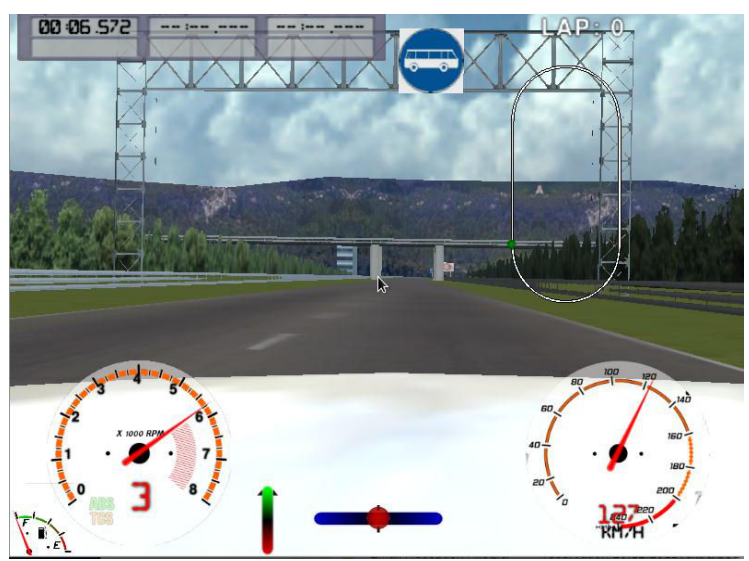

Figure 1. Experimental protocol. A. Subject sitting in the driving simulator. The seat is an electrically adjustable real automobile seat. The pedals and steering wheel are placed in a layout close to a small car's setting B. Visual field showing the simulated Swiss highway

\section{B. Driving data processing}

The simulated highway was shaped as a large oval with two long straight lines and two large curves in order to provide continuous recording over long periods of time. One lap took about $2 \mathrm{~min}$ and for each straight line the subject had to perform a lane change to the left and one to the right. The highway section for lane change was roughly predefined but the subject was allowed to perform the lane changes at any place within these sections in order to study self-paced actions. Figure 2 A presents the whole trajectory of the vehicle for several laps and on the right a detailed view of the straight line segment.

Lane changes and straight driving periods were identified from the trajectory and based on the steering data. The location of lane changes (green and red dots) is similar for each lap but not exactly at the same, indicating self-paced actions.

Since the vehicle was driven at high speed (approx. $120 \mathrm{~km} / \mathrm{h}$ ) only small steering action were possible for the lane changes in order to avoid the vehicle leaving the road. The start point of the steering action was identified from the steering position and further used as the onset of movement (Figure 2B). The periods of straight driving where also evaluated using the steering profile. Periods where the steering activity was smaller than 3 degrees were considered as straight period, thus allowing small oscillations.

A

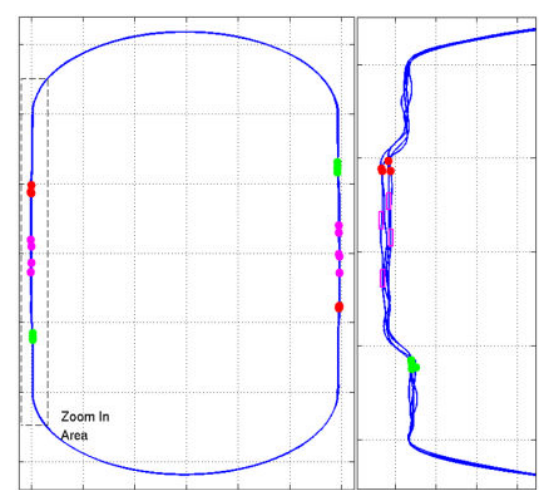

B

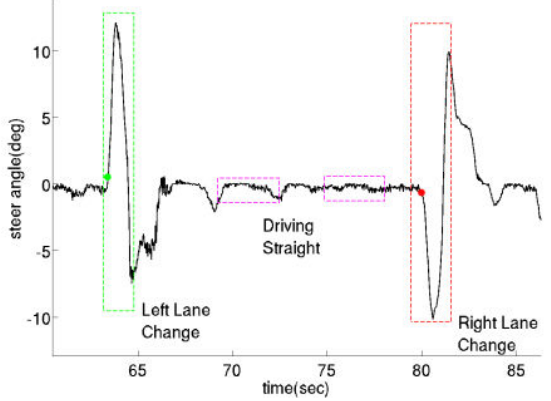

Figure 2. A. Vehicle trajectory on the whole circuit (left) and straight line (right). Red dots are the points where a lane change to the right was detected from the trajectories, green dots where a lane change to the left was detected and magenta rectangles show straight driving periods. B. Steering profile example on the straight line. The green rectangle is the area identified from the trajectory as a left lane change, the red rectangle as a right lane change and magenta rectangles show rather straight trajectories. The dots are the onset of the left steering action (green) and right steering action(red)

\section{EEG Signal Processing}

The EEG signals were filtered between $0.1 \mathrm{~Hz}$ and $1 \mathrm{~Hz}$ using a 4th order Butterworth filter. Next, common average reference (CAR) was used to remove background brain activity [12], and finally, the mean value was subtracted from each channel.

Each time the driver changed lane defined a new trial. Within each trial, three types of epochs were defined: Preparation, Steering and Straight Epoch. Preparation Epochs designate the $4 \mathrm{~s}$ before the start of the steering action and Steering Epochs the $4 \mathrm{~s}$ after the start of the steering. Periods of $4 \mathrm{~s}$ on straight lines with no large steering actions are called Straight Epochs. Note that while the Preparation and Steering epochs are continuous in time, the Straight one is independent. However, the latter was chosen within the same lap and considered as part of the same trial. Even though more Straight epochs could be retrieved, for this study we used only the same amount as the Preparation epochs in order to retrieve a balanced number of epochs. For this study, left and right 
steering actions were considered together, the comparison being performed between the Preparation Epochs and the Straight Epochs. Figure 3 A shows the grand averages for the 3 types of epochs on $\mathrm{Cz}, \mathrm{C} 1$ and $\mathrm{C} 2$ for one subject. The other 5 subjects showed similar trends with some differences in amplitude. On average, there were about 60 epochs of each type per subject. For the preparation trials a negative potential builds up more than $1 \mathrm{~s}$ before the onset of the movement, akin to the reported Motion Related Potential. On the other hand, for the Straight epochs no particular activity appears. The topographic display in Figure $3 \mathrm{~B}$ shows that the negative potential is spread over the motor cortex.

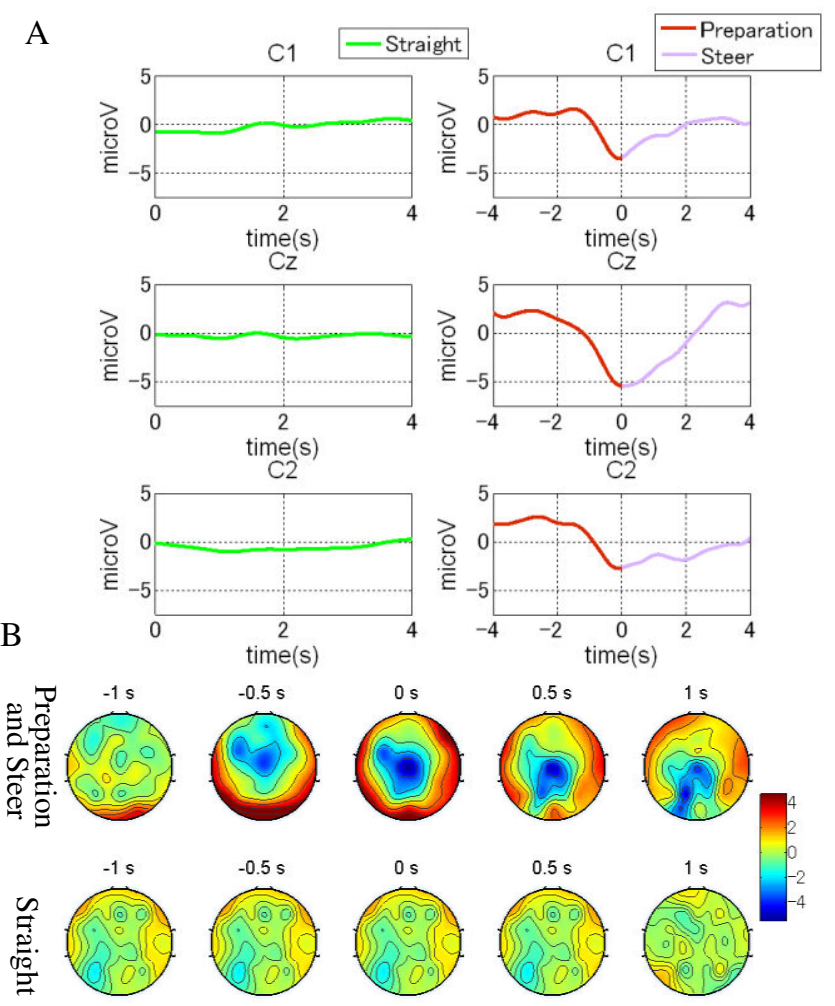

Figure 3. A. EEG averages of $\mathrm{C} 1 \mathrm{Cz} C 2$ for one subject. $\mathrm{t}=0 \mathrm{~s}$ is the onset of the steering action. The grand averages for the Straight trials show no significant time locked activity. In the second half of the Preparation period a negative potential locked on the onset of movement builds up and recovers in the first half of the Steering period B. Topographic representation for one subject. A negative potential time locked on the onset of steering is spread over the motor cortex.

\section{Single Trial Classification}

We assessed offline single trial classification using Linear Discriminant Analysis (LDA) [13]. LDA classifiers were trained on windows of Preparation and Straight trials data. In order to see the influence of the training data position in time, six different time windows were tested: from a window ending at $800 \mathrm{~ms}$ before the onset of the movement, until one ending $300 \mathrm{~ms}$ before movement, in steps of $100 \mathrm{~ms}$. Also two lengths of the window were considered: $250 \mathrm{~ms}$ and $500 \mathrm{~ms}$. In total, for each subject, 12 different classifiers were trained using 48 features: 8 equidistant points within the training window for 6 channels (C1, Cz, C2, CP1, CPz, CP2). The more features one can use the more information is available to differentiate between classes. On the other hand more training data is needed. In this case we chose the number of features based on the estimated number of training samples. Since the number of trials was around 60 a simple method was applied to increase the number of samples used to train the classifiers. Instead of extracting one sample per epoch, three samples were extracted from each epoch. For the first sample, the values for the features were extracted from the window with the predefined length ending at the time of interest. Then, to extract two other samples the window was shifted backward about 70ms at a time, and a new sample was extracted. We chose a shift value such that in the portion where the windows overlap the values for the features are never from the same point of the epoch.

After training, each of the classifiers was applied in a sliding window over the last $2 \mathrm{~s}$ of the epochs. True Positive Rates (TPR) and False Positive Rates (FPR) across trials were calculated at each time point in a 5-fold cross-validation process. Here TPR is calculated as the percentage of test samples from Preparation data that have been correctly classified as Preparation. Similarly, FPR is the percentage of test samples from Straight data that have been wrongly classified as Preparation.

\section{RESULTS}

Figure 4 shows the average TPR and FPR at each time point for the 12 classifiers for one subject (green and magenta lines, respectively). The top row shows classifiers trained on a 250 ms window $(\mathrm{w}=250)$ and the bottom row shows the ones trained on $500 \mathrm{~ms}$ window $(\mathrm{w}=500)$. Each plot represents a different time of interest for which the classifier was trained (e.g., $\mathrm{t}=-0.8$ means that the training window ends $800 \mathrm{~ms}$ before the onset). Chance level has been calculated by randomizing the data and performing the same classification 10 times.

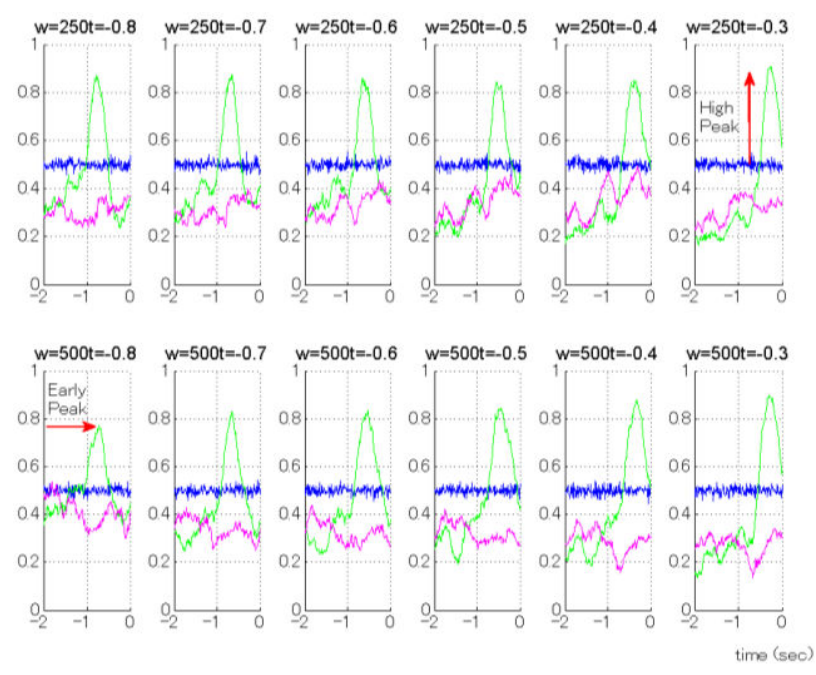

Figure 4. Average TPR and FPR for different training intervals and window lengths for one subject. Blue line is chance level, green line is TPR and magenta is FPR at that time for Preparation Epochs across the 5 folds

While the differences in TPR and FPR between the two window sizes and the 6 training positions are small, there is a 
trade off between detection time and the TPR and FPR. The later the TPR peak, the higher it is and the lower FPR is. This reflects the increase of the negativity closer to the onset of the movement.

We aggregated the results from each subject based on 2 types of criteria

Earliest TPR Peak: the classifier with the earliest peak was chosen for each subject for each window size.

Highest TPR Peak: the classifier with the highest peak was chosen for each subject for each window size.

The average results across all the subjects are presented in Table1

TABLE I. ClasSIFICATION RESULTS

\begin{tabular}{|l|l|l|}
\hline \multirow{2}{*}{ Criteria } & \multicolumn{2}{|c|}{ Classifier Performance } \\
\cline { 2 - 3 } & \multicolumn{1}{|c|}{ Window $=\mathbf{2 5 0} \mathbf{~} \boldsymbol{s}$} & \multicolumn{1}{c|}{ Window=500 $\mathbf{~ s}$} \\
\hline \multirow{2}{*}{ Earliest Peak } & $\mathrm{t}=-811 \pm 46 \mathrm{~ms}$ & $\mathrm{t}=-760 \pm 31 \mathrm{~ms}$ \\
& $\mathrm{TPR}=74.65 \pm 8.2 \%$ & $\mathrm{TPR}=74.9 \pm 7.9 \%$ \\
\hline \multirow{2}{*}{ Highest Peak } & $\mathrm{t}=-430 \pm 220 \mathrm{~ms}$ & $\mathrm{t}=-613 \pm 169 \mathrm{~ms}$ \\
& $\mathrm{TPR}=76.9 \pm 9.3 \%$ & $\mathrm{TPR}=79.4 \pm 9.2 \%$ \\
\hline
\end{tabular}

To better understand the differences between the 2 training windows and between the 2 criteria, False Positive rates were also taken into account. The results for all 6 subjects for the chosen classifiers are presented in Figure 5. In terms of predictive power the differences between the 4 classifier groups are small. This means that even using a shorter training window $(250 \mathrm{~ms})$ can yield similar predictive power with the larger one $(500 \mathrm{~ms})$. Also, in terms of predictive power, Earlier Peaking classifiers perform similarly to Higher Peaking classifiers.

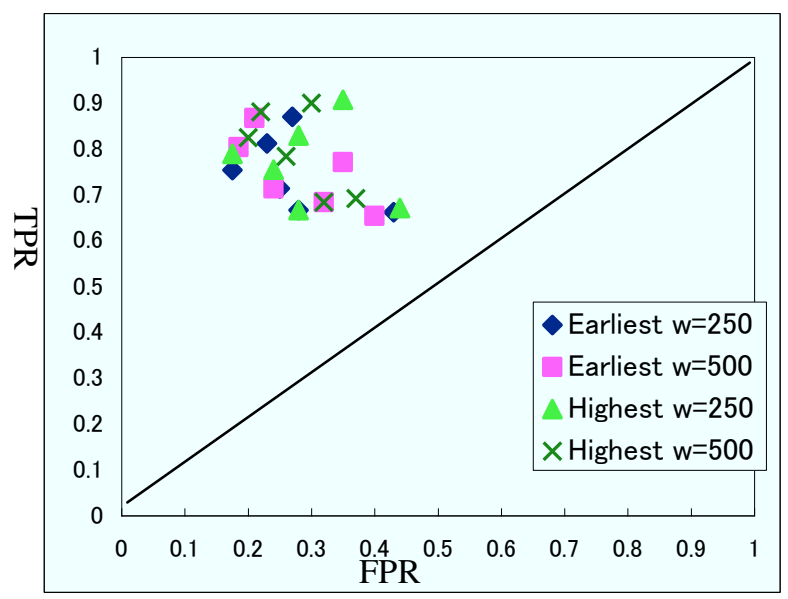

Figure 5. Classifiers performance in ROC space. Closer to top left corner means better performance

\section{CONCLUSIONS AND DISCUSSION}

Aiming at predicting driver's steering actions, we analyzed the brain activity correlates to upper limb movements in a simple lane change task. Firstly, we were able to confirm the presence of Motion Related Potentials time locked to the onset of movement, in this case steering. Secondly, we assessed the offline single trial classification with encouraging results. It is important to stress that the data was recorded during driving which means that the subject was not only performing arm movements as in previous in lab studies, but was involved in several cognitive processes such as attending to the continuous visual input and controlling the vehicle. Considering this, the fact that our classifiers were able to predict the onset of steering about $800 \mathrm{~ms}$ earlier with a TPR of approx. $74.6 \%$ is rather promising. We have also seen that there are no large differences when using a shorter window for classification which leaves the door open to lower computational means.

A natural line for future works is to try to classify not only the onset of the movement but also the direction of the movement. Another line is obviously to come up with an online detection method and to develop a real interaction method. Having such a system in place is also essential to answer another open key question: what is the required minimal accuracy for such a system for the driver to feel comfortable in the interaction?

\section{ACKNOWLEDGMENT}

We would like to thank all subjects that have participated in the experiments spending long time in a simple repetitive task.

\section{REFERENCES}

[1] J. d. R. Millán, P. W. Ferrez, F. Galán, E. Lew, and R. Chavarriaga, “ Non-invasive brain-machine interaction," in Int J Pattern Recognition and Artificial Intelligence, 2007,vol. 22, pp. 959-972,

[2] C. H. Chuang, P. C. Lai, L. W. Ko, B. C. Kuo, and C. T. Lin,"Driver's cognitive state classification toward brain computer interface via using a generalized and supervised technology," in IJCNN, 2010, pp. 1-7

[3] F. C. Lin, L. W. Ko, S. Chen, C. Chen, and C. Lin, "EEG based cognitive state monitoring and prediction by using the self constructing neural fuzzy system," in ISCAS, 2010, pp. 2287-2290

[4] K. Itoh, Y. Miki, N. Kubo, Y. Takeda, H. Tanaka, "A Study on Estimation the Variation of Driver's State by EEGs and EOGs", in SAE, 2006.

[5] Y. Kobayashi, T. Kimura, T. Yamamura, G. Naito et al., "Development of a Prototype Driver Support System With Accelerator Pedal Reaction Force Control and Driving and Braking Force Control, " in SAE 2006

[6] L. Gheorghe and T. Sunda, "Brain Waves Measurement Based Evaluation of Mental Workload Related to Visual Information While Driving, " in SAE, 2011

[7] E. Lew, R. Chavarriaga, S. Silvoni, J. d. R. Millán, "Detection of self-paced reaching movement intention from EEG signals," in Frontiers in Neuroengineering, 2012, Volume 5, 2-17.

[8] S. Haufe, M. S. Treder, M. F. Gugler, M. Sagebaum, G. Curio, B. Blankertz, "EEG potentials predict upcoming emergency brakings during simulated driving", in Journal of Neural Engineering, vol. 8, no. 5, pp. 1-11, 2011.

[9] H.H. Kornhuber and L. Deecke, "Changes in the brain potential in voluntary movements and passive movements in man: readiness potentials and reafferent potentials," in PflugersArch.Gesamte Physiol. MenschenTiere, 1965, vol. 284, pp. 1-17.

[10] B. Libet, C.A. Gleason, E. W. Wright, and D.K. Pearl, "Time of conscious intention to act in relation to onset of cerebral activity (readiness-potential) ," Brain, 1983, vol. 106, pp. 623-642.

[11] B. Libet, E.W. Wright, and C. A. Gleason, "Readiness-potentials preceding unrestricted spontaneous' vs. pre-planned voluntary acts, " in Electroencephalography and Clinical Neurophysiology, 1982, vol. 54, pp. 322-335

[12] D. McFarland, L. McCane, S. David, and J. Wolpaw, "Spatial filter selection for EEG-based communication," in Electroencephalography and Clinical Neurophysiology, 1997, vol. 103, no. 3, pp. 386-394,

[13] R. O. Duda, P. E. Hart, and D. G. Stork, "Pattern Classification, 2nd ed., " in New York: Wiley, 2001. 